\title{
Portal Vein Thrombosis after Percutaneous Liver Biopsy
}

\author{
Jerome Dumortier, Sabine Ficarelli, Olivier Boillot
}

1) Hôpital Edouard Herriot, Pavillon L, Fédération des Spécialités Digestives, Lyon, France
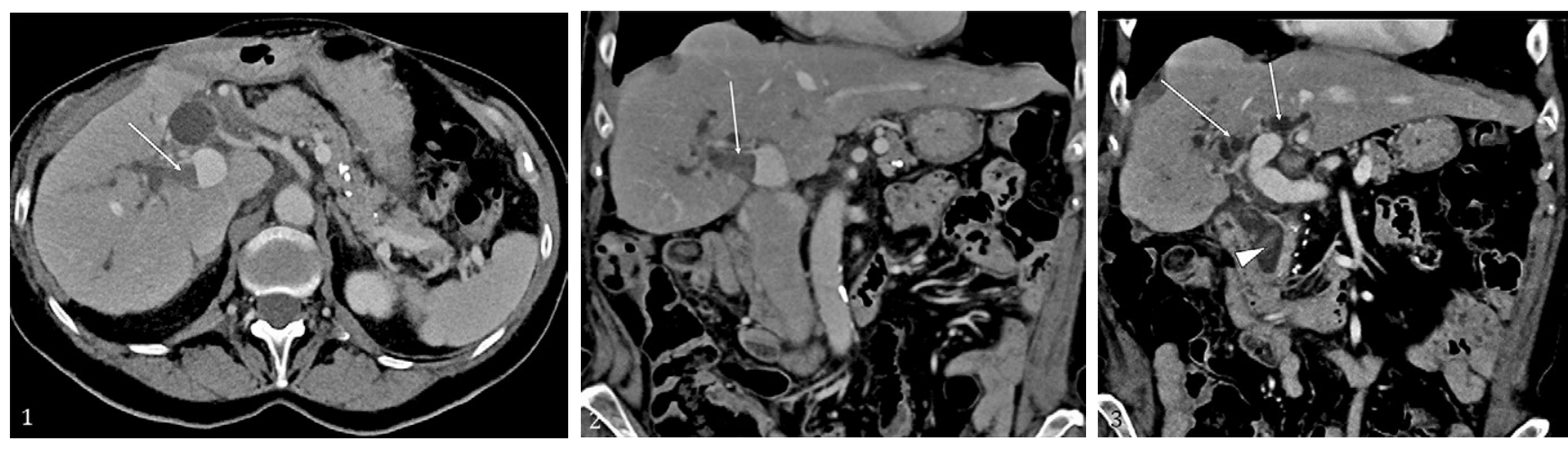

A 72-year-old woman, with a history of liver transplantation in 1991 because of fulminant hepatitis underwent a protocol percutaneous liver biopsy in March 2021. Computed tomography (CT) scan before liver biopsy was normal. The patient was admitted five days after liver biopsy because of abdominal pain. A new CT scan with contrast agent disclosed a thrombosis of the right portal vein in the portal phase (Fig. 1 transversal section, 2 coronal section), associated with biliary ducts enlargement (Fig. 3, coronal section, arrows) and probable haemobilia (Fig. 3, arrowhead). Hemoglobin level remained stable. Radiological follow-up one week later confirmed persistent portal vein thrombosis, with partial resolution of biliary abnormalities; anticoagulation treatment was started.

Common complications of liver biopsy include pain and bleeding (hemoperitoneum, intrahepatic hematoma, haemobilia, hemothorax) and can be severe [1]. The mortality rate is less than $0.4 \%$ [2], but complications of liver biopsy can lead to additional testing, procedures, treatments and hospitalizations. Portal vein thrombosis appears as very exceptional since only one case has been reported [3]. In this case, the authors suspected that the cause of the thrombus was damage to a small branch of portal vein during biopsy, given the location of the thrombus. We report here the first case of portal vein thrombosis associated with haemobilia, and we hypothesize that thrombosis was due to an iatrogenic acute porto-biliary fistula.

Corresponding author: Prof. Jerome Dumortier, jerome.dumortier@chu-lyon.fr

Conflicts of interest: None to declare.

\section{REFERENCES}

1. Piccinino F, Sagnelli E, Pasquale G, Giusti G. Complications following percutaneous liver biopsy. A multicentre retrospective study on 68,276 biopsies. J Hepatol 1986;2:165-173. doi:10.1016/s0168-8278(86)80075-7

2. Rockey DC, Caldwell SH, Goodman ZD, Nelson RC, Smith AD; American Association for the Study of Liver Diseases. Liver biopsy. Hepatology 2009;49:1017-1044. doi:10.1002/hep.22742

3. Boschuetz N, Gray M, Bari K. Portal Vein Thrombosis After Percutaneous Liver Biopsy. Clin Gastroenterol Hepatol 2018;16:e5-e6. doi:10.1016/j.cgh.2017.07.027 